\title{
Rodé's theorem on common fixed points of semigroup of nonexpansive mappings in $\operatorname{CAT}(0)$ spaces
}

\author{
Watcharapong Anakkamatee ${ }^{1}$ and Sompong Dhompongsa ${ }^{1,2^{*}}$
}

\author{
* Correspondence: \\ sompongd@chiangmai.ac.th \\ 'Department of Mathematics, \\ Faculty of Science, Chiang Mai \\ University, Chiang Mai, 50200, \\ Thailand \\ Full list of author information is \\ available at the end of the article
}

\author{
Abstract \\ We extend Rodé's theorem on common fixed points of semigroups of nonexpansive \\ mappings in Hilbert spaces to the CAT(0) space setting. \\ 2000 Mathematics Subject Classification: 47H09; 47H10.
}

Keywords: CAT(0) space, semigroup of nonexpansive mappings, $\Delta$ ? $\triangle$ ?-convergence

\section{Introduction}

In 1976, Lim [1] introduced a concept of convergence in a general metric space, called strong $\Delta$-convergence. In [2], Kirk and Panyanak introduced a concept of convergence in a $\mathrm{CAT}(0)$ space, called $\Delta$-convergence (see Section 2 for the definition). Moreover, they showed that many Banach space concepts and results which involve weak convergence can be extended to the $\mathrm{CAT}(0)$ space setting by using the $\Delta$-convergence.

For each semigroup $S$, let $B(S)$ be the Banach space of all bounded real-valued mappings on $S$ with supremum norm. A continuous linear functional $\mu \in B(S)^{*}$ (the dual space of $B(S))$ is called a mean on $B(S)$ if $\|\mu\|=\mu(1)$. For any $f \in B(S)$, we use the following notation:

$$
\mu(f)=\mu_{s}(f(s)) .
$$

A mean $\mu$ on $B(S)$ is said to be left invariant [respectively, right invariant] if $\mu_{s}(f(t s))$ $=\mu_{s}(f(s))$ [respectively, $\mu_{s}(f(s t))=\mu_{s}(f(s))$ ] for all $f \in B(S)$ and for all $t \in S$. We will say that $\mu$ is an invariant mean if it is both left and right invariants. If $B(S)$ has an invariant mean, we call $S$ an amenable semigroup. It is well known that every commutative semigroup is amenable [3]. For each $s \in S$ and $f \in B(S)$, we define elements $l_{s} f$ and $r_{s} f$ in $B(S)$ by $\left(l_{s} f\right)(t)=f(s t)$ and $\left(r_{s} f\right)(t)=f(t s)$ for any $t \in S$, respectively. A net $\left\{\mu_{\alpha}\right\}$ of means on $B(S)$ is said to be asymptotically invariant if

$$
\lim _{\alpha}\left(\mu_{\alpha}\left(l_{s} f\right)-\mu_{\alpha}(f)\right)=0=\lim _{\alpha}\left(\mu_{\alpha}\left(r_{s} f\right)-\mu_{\alpha}(f)\right) .
$$

In [4], Rodé proved the following:

Theorem 1.1. [4] If $S$ is an amenable semigroup, $C$ is a closed convex subset of a Hilbert space $H, \mathcal{S}=\left\{T_{s}: s \in S\right\}$ is a nonexpansive semigroup on $C$ such that the set $F(\mathcal{S})$ of common fixed points of Sis nonempty and $\left\{\mu_{\alpha}\right\}$ is an asymptotically invariant net of means, then for each $x \in C,\left\{T_{\mu_{\alpha}} x\right\}$ converges weakly to an element of $F(\mathcal{S})$.

(C) 2011 Anakkamatee and Dhompongsa; licensee Springer. This is an Open Access article distributed under the terms of the Creative Commons Attribution License (http://creativecommons.org/licenses/by/2.0), which permits unrestricted use, distribution, and reproduction in any medium, provided the original work is properly cited. 
Further, for each $x \in C$, the limit point of $\left\{T_{\mu_{\alpha}} x\right\}$ is the same for all asymptotically invariant nets of means $\left\{\mu_{\alpha}\right\}$.

It is remarked that if $S$ is amenable, then there is always an asymptotically strong invariant net of finite means, i.e., means that are convex combination of point evaluations. This follows from Proposition 3.3 in [5].

Development of this subject had been made by several authors [1,6-8]. The main purpose of this article is to extend this result of Rodé for a nonexpansive semigroup on a $\mathrm{CAT}(0)$ space in which the $\Delta$-convergence plays the role of weak convergence.

\section{Preliminaries}

Let $(X, d)$ be a metric space. A geodesic joining $x \in X$ to $y \in X$ is a mapping $c$ from a closed interval $[0, l] \subset \mathbb{R}$ to $X$ such that $c(0)=x, c(l)=y$ and $d\left(c(t), c\left(t^{\prime}\right)\right)=\left|t-t^{\prime}\right|$ for all $t, t^{\prime} \in[0, l]$. In particular, $c$ is an isometry and $d(x, y)=l$. The image $\gamma$ of $c$ is called a geodesic (or metric) segment joining $x$ and $y$. When it is unique, this geodesic is denoted $[x, y]$. Write $c(\alpha 0+(1-\alpha) l)=\alpha x \oplus(1-\alpha) y$, and for $\alpha=\frac{1}{2}$, we write $\frac{1}{2} x \oplus \frac{1}{2} y$ as $\frac{x \oplus y}{2}$, the midpoint of $x$ and $y$. The space $X$ is said to be a geodesic space if every two points of $X$ are joined by a geodesic.

Following [2], a metric space $X$ is said to be a $C A T(0)$ space if it is geodesically connected and if every geodesic triangle in $X$ is at least as thin as its comparison triangle in the Euclidean plane. This latter property, which is what we referred to as the $(\mathrm{CN})$ inequality, enables one to define the concept of nonpositive curvature in this situation, generalizing the same concept in Riemannian geometry. In fact (cf. [[9], p. 163]), the following are equivalent for a geodesic space $X$ :

(i) $X$ is a $\operatorname{CAT}(0)$ space.

(ii) $X$ satisfies the $(\mathrm{CN})$ inequality: If $x_{0}, x_{1} \in X$ and $\frac{x_{0} \oplus x_{1}}{2}$ is the midpoint of $x_{0}$ and $x_{1}$, then

$$
d^{2}\left(y, \frac{x_{0} \oplus x_{1}}{2}\right) \leq \frac{1}{2} d^{2}\left(y, x_{0}\right)+\frac{1}{2} d^{2}\left(y, x_{1}\right)-\frac{1}{4} d^{2}\left(x_{0}, x_{1}\right), \text { for all } y \in X .
$$

(iii) $X$ satisfies the Law of cosine: If $a=d(p, q), b=d(p, r), c=d(q, r)$ and $\xi$ is the Alexandrov angle at $p$ between $[p, q]$ and $[p, r]$, then $c^{2} \geq a^{2}+b^{2}-2 a b \cos \xi$.

For any subset $C$ of $X$, let $\pi=\pi_{D}$ be a nearest point projection mapping from $C$ to a subset $D$. It is known by [[9], pp. 176-177] (see also [[10], Proposition 2.6]) that if $D$ is closed and convex, the mapping $\pi$ is well-defined, nonexpansive, and satisfies

$$
d^{2}(x, y) \geq d^{2}(x, \pi x)+d^{2}(\pi x, y) \text { for all } x \in C \text { and } y \in D .
$$

Definition 2.1. [[11], Definition 5.13] A complete CAT(0) space $X$ has the property of the nice projection onto geodesics (property $(N)$ for short) if, given any geodesic segment $I \subset X$, it is the case that $\pi_{I}(m) \in\left[\pi_{I}(x), \pi_{I}(y)\right]$ for any $x, y$ in $X$ and $m \in[x, y]$.

As noted in [11], we do not know of any example of a CAT $(\kappa)$ space which does not enjoy the property $(\mathrm{N})$. 
Let $S$ be a semigroup, $C$ be a closed convex subset of a Hilbert space $H$, and for each $s$ in $S, T_{s}$ is a mapping from $C$ into itself. Suppose $\left\{T_{s} x: s \in S\right\}$ is bounded for all $x \in$ $C$. Let $x \in C$ and $\mu$ be a mean on $B(S)$. By Riesz's representation theorem, there exists a unique $x_{0} \in C$ such that

$$
\mu_{s}\left\langle T_{s} x, y\right\rangle=\left\langle x_{0}, y\right\rangle
$$

for all $y \in H$. Here $\langle$,$\rangle denotes the inner product on H$.

The following result is a mild generalization of a result of Kakavandi and Amini [[12], Lemma 2.1].

Lemma 2.2. Let $C$ be a closed convex subset of a $C A T(0)$ space $X$ and $\mu$ be a mean on $B(S)$. For a bounded function $h: S \rightarrow C$, define

$$
\varphi_{\mu}(\gamma):=\mu_{s}\left(d^{2}(h(s), y)\right)
$$

for all $y \in X$. Then, $\phi_{\mu}$ attains its unique minimum at a point of $\overline{c o}\{h(s): s \in S\}$.

For each $x \in C$, denote $\mathcal{S}(x):=\left\{T_{s} x: s \in S\right\}$. If $\mathcal{S}(x)$ is bounded, then by Lemma 2.2 we put

$$
T_{\mu}(h):=\operatorname{argmin}\left\{y \mapsto \mu_{s}\left(d^{2}(h(s), y)\right)\right\},
$$

and for $h(s)$ of the form $T_{s} x$, we write $T_{\mu}(h)$ shortly as $T_{\mu} x$.

Remark 2.3. If $X$ is a Hilbert space, then

(i) $T_{\mu} x=x_{0}$ where $x_{0}$ verifies (2), and

(ii) $\left\|x_{0}\right\|^{2}=\sup _{y \in X}\left(2\left\langle x_{0}, y\right\rangle-\|y\|^{2}\right)$.

Proof. (i): Let $x_{0}$ be such that $\mu_{s}\left\langle T_{s} x, y\right\rangle=\left\langle x_{0}, y\right\rangle$ for all $y \in X$. We have $\phi_{\mu}\left(x_{0}\right)=\phi_{\mu}(0)$ $+\left\|x_{0}\right\|^{2}-2\left\langle x_{0}, x_{0}\right\rangle=\phi_{\mu}(0)-\left\|x_{0}\right\|^{2} \leq \phi_{\mu}(0)+\left\|T_{\mu} x\right\|^{2}-2\left\langle x_{0}, T_{\mu} x\right\rangle=\phi_{\mu}\left(T_{\mu} x\right)$. Therefore, $x_{0}=T_{\mu} x$.

(ii): For any $x, y \in X$, we know that $\left\|T_{s} x-y\right\|^{2}=\left\|T_{s} x\right\|^{2}-2\left\langle T_{s} x, y\right\rangle+\|y\|^{2}$. By the linearity of $\mu$ and (2), we have $\mu_{s}\left(\left\|T_{s} x-y\right\|^{2}\right)=\mu_{s}\left(\left\|T_{s} x\right\|^{2}\right)-2\left\langle x_{0}, y\right\rangle+\|y\|^{2}$. Thus, $\inf _{y \in X} \mu_{s}\left(\left\|T_{s} x-y\right\|^{2}\right)=\mu_{s}\left(\left\|T_{s} x\right\|^{2}\right)-\sup _{y \in X}\left(2\left\langle x_{0}, y\right\rangle-\|y\|^{2}\right)$. On the other hand, by (i), $\inf _{y \in X} \mu_{s}\left(\left\|T_{s} x-y\right\|^{2}\right)=\mu_{s}\left(\left\|T_{s} x-x_{0}\right\|^{2}\right)=\mu_{s}\left(\left\|T_{s} x\right\|^{2}\right)-2 \mu_{s}\left\langle T_{s} x, x_{0}\right\rangle+\left\|x_{0}\right\|^{2}=\mu_{s}(\|$ $\left.T_{s} x \|^{2}\right)-\left\|x_{0}\right\|^{2}$. Hence, $\left\|x_{0}\right\|^{2}=\sup _{y \in X}\left(2\left\langle x_{0}, y\right\rangle-\|y\|^{2}\right)$.

Let $C$ be a closed convex subset of a $\operatorname{CAT}(0)$ space $X$ and $S$ a semigroup. We say that the set $\mathcal{S}(S):=\left\{T_{s}: s \in S\right\}$ is a nonexpansive semigroup on $C$ if

(i) $T_{s}: C \rightarrow C$ is a nonexpansive mapping, i.e., $d\left(T_{s} x, T_{s} y\right) \leq d(x, y)$ for all $x, y \in X$, for all $s \in S$,

(ii) the mapping $s \rightarrow T_{s} x$ is bounded for all $x \in C$, and

(iii) $T_{t s}=T_{t} T_{s}$, for all $s, t \in S$.

We denote by $F(\mathcal{S})$ the set of all common fixed points of mappings in $\mathcal{S}(S)$, i.e., $F(\mathcal{S}):=\bigcup_{s \in S} F\left(T_{s}\right)$, where $F\left(T_{s}\right):=\left\{x \in C: T_{s} x=x\right\}$ is the set of fixed points of $T_{s}$.

For any bounded net $\left\{x_{\alpha}\right\}$ in a closed convex subset $C$ of a $C A T(0)$ space $X$, put

$$
r\left(x,\left\{x_{\alpha}\right\}\right)=\limsup d\left(x, x_{\alpha}\right)
$$


for each $x \in C$. The asymptotic radius of $\left\{x_{\alpha}\right\}$ on $C$ is given by

$$
r\left(C,\left\{x_{\alpha}\right\}\right)=\inf _{x \in C} r\left(x,\left\{x_{\alpha}\right\}\right),
$$

and the asymptotic center of $\left\{x_{\alpha}\right\}$ in $C$ is the set

$$
A\left(C,\left\{x_{\alpha}\right\}\right)=\left\{x \in C: r\left(x,\left\{x_{\alpha}\right\}\right)=r\left(C,\left\{x_{\alpha}\right\}\right)\right\} .
$$

It is known that in a complete $\mathrm{CAT}(0)$ space, $A\left(C,\left\{x_{\alpha}\right\}\right)$ consists of exactly one point and $A\left(X,\left\{x_{\alpha}\right\}\right)=A\left(C,\left\{x_{\alpha}\right\}\right)$ (cf. [2]).

Remark 2.4. (i) Let $D, E$ be directions and $v: E \rightarrow D$. If $\left\{x_{v(\beta)}: \beta \in E\right\}$ is a subnet of a bounded net $\left\{x_{\alpha}: \alpha \in D\right\}$, then $r\left(C,\left\{x_{v(\beta)}\right\}\right) \leq r\left(C,\left\{x_{\alpha}\right\}\right)$.

(ii) Let $C$ be a closed convex subset of a $C A T(0)$ space $X, T: C \rightarrow C$ a nonexpansive mapping and $x \in C$. If $\left\{T^{n} x\right\}$ is bounded and if $z \in A\left(C,\left\{T^{n} x\right\}\right)$, then $z \in F(T)$.

Proof. (i) Let $\alpha_{0} \in D$. By the definition of subnets, there exists $\beta_{0} \in E$ such that $v(\beta)$ $\geqslant \alpha_{0}$ for all $\beta \geqslant \beta_{0}$. For each $x \in C$, we have $\sup _{\alpha \succcurlyeq \alpha_{0}} d\left(x, x_{\alpha}\right) \geq \sup _{\beta \succcurlyeq \beta_{0}} d\left(x, x_{v(\beta)}\right)$. Thus, $\sup _{\alpha \succcurlyeq \alpha_{0}} d\left(x, x_{\alpha}\right) \geq \inf _{\beta_{1}} \sup _{\beta \succcurlyeq \beta_{1}} d\left(x, x_{v(\beta)}\right)$, and this holds for all $\alpha_{0}$. Hence, $r\left(x,\left\{x_{\alpha}\right\}\right)=\inf _{\alpha_{0}} \sup _{\alpha \succcurlyeq \alpha_{0}} d\left(x, x_{\alpha}\right) \geq r\left(x, x_{v(\beta)}\right)$, and this holds for all $x \in C$. Consequently, $r\left(C,\left\{x_{\alpha}\right\}\right)=\inf _{x \in C} r\left(x,\left\{x_{\alpha}\right\}\right) \geq \inf _{x \in C} r\left(x, x_{v(\beta)}\right)=r\left(C,\left\{x_{v(\beta)}\right\}\right)$.

(ii) Since $T$ is nonexpansive, $\lim \sup _{n} d^{2}\left(T^{n} x, T z\right)=\lim \sup _{n} d^{2}\left(T T^{n} x, T z\right) \leq \lim \sup _{n}$ $d^{2}\left(T^{n} x, z\right)$.

As every asymptotic center is unique, we have $z=T z$. $\square$

Definition 2.5. [[2], Definition 3.3] $A$ net $\left\{x_{\alpha}\right\}$ in $X$ is said to $\Delta$-converge to $x \in X$ if $x$ is the unique asymptotic center of $\left\{u_{\beta}\right\}$ for every subnet $\left\{u_{\beta}\right\}$ of $\left\{x_{\alpha}\right\}$. In this case, we write $\Delta-\lim _{\alpha} x_{\alpha}=x$ and call $x$ the $\Delta$-limit of $\left\{x_{\alpha}\right\}$.

Proposition 2.6. [[2], Proposition 3.4] Every bounded net in $X$ has a $\Delta$-convergent subnet.

Remark 2.7. (i) Let $D$ be a direction, $\left\{x_{\alpha}: \alpha \in D\right\}$ a net in $X$ and $x \in X$. If $\lim \sup _{\alpha}$ $d\left(x, x_{\alpha}\right)>\rho$ for some $\rho>0$, then there exists a subnet $\left\{x_{\beta_{\alpha}}\right\}$ of $\left\{x_{\alpha}\right\}$ such that $d\left(x, x_{\beta_{\alpha}}\right) \geq \rho$ for all $\alpha$.

(ii) Let $\left\{x_{\alpha}\right\}$ be a net in $X$. Then, $\left\{x_{\alpha}\right\} \Delta$-converges to $x \in X$ if and only if every subnet $\left\{x_{\alpha^{\prime}}\right\}$ of $\left\{x_{\alpha}\right\}$ has a subnet $\left\{x_{\alpha^{\prime \prime}}\right\}$ which $\Delta$-converges to $x$.

Proof. (i): For each $\alpha \in D$, we have $\sup _{\alpha^{\prime} \geqslant \alpha} d\left(x, x_{\alpha^{\prime}}\right)>\rho$. Thus there exists $\beta_{\alpha}>\alpha$ such that $d\left(x, x_{\beta_{\alpha}}\right) \geq \rho$, and this holds for all $\alpha$. Set a set $E=\left\{\beta_{\alpha}: \alpha \in D\right\}$. Clearly, $E$ is a direction, and define $v: E \rightarrow D$ by $v\left(\beta_{\alpha}\right)=\beta_{\alpha}$. Let $\alpha_{0} \in D$, thus $v\left(\beta_{\alpha}\right) \geqslant \alpha_{0}$ for all $\beta_{\alpha} \succcurlyeq \beta_{\alpha_{0}}$ and this shows that $\left\{x_{\beta_{\alpha}}\right\}$ is a subnet of $\left\{x_{\alpha}\right\}$ satisfying $d\left(x, x_{\beta_{\alpha}}\right) \geq \rho$ for all $\alpha$.

ii): It is easy to see that if $\left\{x_{\alpha}\right\} \Delta$-converges to $x$, then every subnet of $\left\{x_{\alpha}\right\}$ also $\Delta$-converges to $x$. On the other hand, suppose $\left\{x_{\alpha}\right\}$ does not $\Delta$-converge to $x$. Thus, there exists a subnet $\left\{x_{\beta}\right\}$ of $\left\{x_{\alpha}\right\}$ such that $x \notin A\left(C,\left\{x_{\beta}\right\}\right)$, and so $\lim \sup _{\beta} d\left(x, x_{\beta}\right)>\rho$ $>r\left(C,\left\{x_{\beta}\right\}\right)$ for some $\rho>0$. By (i), there exists a subnet $\left\{x_{\gamma_{\beta}}\right\}$ of $\left\{x_{\beta}\right\}$ satisfying $d\left(x, x_{\gamma_{\beta}}\right) \geq \rho$ for all $\beta$. By assumption, there exists a subnet $\left\{x_{\left(\gamma_{\beta}\right)_{\eta}}\right\}$ of $\left\{x_{\gamma_{\beta}}\right\} \Delta$-converging to $x$. Using Remark $\rho \leq \lim \sup _{\gamma} d\left(x, x_{\left(\gamma_{\beta}\right)_{\eta}}\right)=r\left(C,\left\{x_{\left(\gamma_{\beta}\right)_{\eta}}\right\}\right) \leq r\left(C,\left\{x_{\gamma_{\beta}}\right\}\right) \leq r\left(C,\left\{x_{\beta}\right\}\right)$, a contradiction. $\square$

In [13], Berg and Nikolaev introduced a concept of quasilinearization. Let us formally denote a pair $(a, b) \in X \times X$ by $\overrightarrow{a b}$ and call it a vector. Then, quasilinearization is defined as a map $\langle\rangle:,(X \times X) \times(X \times X)$ by 


$$
\langle\overrightarrow{a b}, \overrightarrow{c d}\rangle=\frac{1}{2} d^{2}(a, d)+\frac{1}{2} d^{2}(b, c)-\frac{1}{2} d^{2}(a, c)-\frac{1}{2} d^{2}(b, d)
$$

for all $a, b, c, d \in X$. Recently, Kakavandi and Amini [14] introduced a concept of wconvergence: a sequence $\left\{x_{n}\right\}$ is said to $w$-converge to $x \in X$ if $\lim _{n \rightarrow \infty}\left\langle\overrightarrow{x_{n} x}, \overrightarrow{a b}\right\rangle=0$ for all $a, b \in X$.

Proposition 2.8. [[14], Proposition 2.5] For sequences in a complete CAT(0) space $X$, $w$-convergence implies $\Delta$-convergence (to the same limit).

A simple example shows that the converse of this proposition does not hold:

Example 2.9. Consider an $\mathbb{R}$-tree in $\mathbb{R}^{\infty}$ defined as follow: Let $\left\{e_{n}\right\}$ be the standard basis, $x_{0}=e_{1}=(1,0,0,0, \ldots)$, and for each $n$, let $x_{n}=x_{0}+e_{n+1}$. An $\mathbb{R}$-tree is formed by the segments $\left[x_{1}, x_{n}\right]$ for $n \geq 0$. It is easy to see that $\left\{x_{n}\right\} \Delta$-converges to $x_{1}$. But $\left\{x_{n}\right\}$ does not $w$-converge to $x_{1}$ since $\left\langle\overrightarrow{x_{n} x_{1}}, \overrightarrow{x_{0} x_{1}}\right\rangle=-1$ for all $n \geq 2$.

Thus, a bounded sequence does not necessary contain an w-convergent subsequence.

\section{Main results}

\section{1 $\Delta$-convergence}

Lemma 3.1. [[12], Lemma 3.1] If $C$ is a closed convex subset of a $C A T(0)$ space $X$ and $T: C \rightarrow C$ is a nonexpansive mapping, then $F(T)$ is closed and convex.

Lemma 3.2. [[12], Proposition 3.2] Let $C$ be a closed convex subset of a $C A T(0)$ space $X$ and $S$ an amenable semigroup. If $\mathcal{S}(S)$ is a nonexpansive semigroup on $C$, then the following conditions are equivalent.

(i) $\mathcal{S}(x)$ is bounded for some $x \in C$;

(ii) $\mathcal{S}(x)$ is bounded for all $x \in C$;

(iii) $F(\mathcal{S}) \neq \emptyset$.

Proposition 3.3. [[12], Theorem 3.3] Let C be a closed convex subset of a complete $C A T(0)$ space $X, S$ an amenable semigroup, and $\mathcal{S}(S)$ a nonexpansive semigroup on $C$ with $F(\mathcal{S}) \neq \emptyset$. Then, $T_{\mu} x \in F(\mathcal{S})$ for any invariant mean $\mu$ on $B(S)$.

We now let $S$ be a commutative semigroup and define a partial order $\geqslant$ on $S$ by $s \geqslant t$ if $s=t$ or there exists $u \in S$ such that $s=u t$. When $s \geqslant t$ but $s \neq t$, we simply write $s \succ$ $t$. We can see that $(S, \geqslant)$ is a directed set. Examples of such $S$ are the usual ordered sets $(\mathbb{N} \cup\{0\},+, \geq)$ and $\left(\mathbb{R}^{+} \cup\{0\},+, \geq\right)$. The following fact is well known:

Proposition 3.4. Let $\mu$ be a right invariant mean on $B(S)$. Then,

$$
\sup _{s} \inf _{t} f(t s) \leq \mu(f(s)) \leq \inf _{s} \sup _{t} f(t s)
$$

for each $f \in B(S)$. Similarly, let $\mu$ be a left invariant mean on $B(S)$. Then,

$$
\sup _{s} \inf _{t} f(s t) \leq \mu(f(s)) \leq \inf _{s} \sup _{t} f(s t)
$$

for each $f \in B(S)$.

Remark 3.5. If $\lim _{s} f(s)=a$ for some $a \in \mathbb{R}$ and $\left\{s^{\prime}\right\}$ is a subnet of $\{s\}$ satisfying $s^{\prime}>s$ for each $s$, then

$$
\mu_{s^{\prime}}\left(f\left(s^{\prime}\right)\right)=a .
$$


Proof. This is an easy consequence of Proposition 3.4 since $\mu_{s^{\prime}}\left(f\left(s^{\prime}\right)\right)=\mu_{s}\left(f\left(s^{\prime}\right)\right)=$ $\lim _{s} f\left(s^{\prime}\right)=a$.

Proposition 3.6. [[12], Proposition 4.1] Let $C$ be a closed convex subset of a complete $C A T(0)$ space $X, S$ a commutative semigroup, and $\mathcal{S}(S)$ a nonexpansive semigroup on $C$ with $F(\mathcal{S}) \neq \emptyset$. Then, for each $x \in C$, the net $\left\{\pi T_{s} x\right\}_{s \in S}$ converges to a point Px in $F(\mathcal{S})$, where $\pi=\pi_{F(\mathcal{S})}: C \rightarrow F(\mathcal{S})$ is the nearest point projection.

Proposition 3.7. Let $C$ be a closed convex subset of a complete CAT(0) space $X, S$ a commutative semigroup, and $\mathcal{S}(S)$ a nonexpansive semigroup on $C$ with $F(\mathcal{S}) \neq \emptyset$. Then, for any invariant mean $\mu$ on $B(S), T_{\mu} x=\lim _{s} \pi T_{s} x=P x$ for all $x \in C$.

Proof. Fix $x \in C$ and let $\varepsilon>0$. From Proposition 3.6, we see that there exists $s_{0} \in S$ such that $d\left(\pi T_{s} x, P x\right)<\varepsilon$ for all $s \geqslant s_{0}$. We know by Proposition 3.3 that $T_{\mu} x \in F(\mathcal{S})$. So, $d\left(P x, T_{s} x\right) \leq d\left(P x, \pi T_{s} x\right)+d\left(\pi T_{s} x, T_{s} x\right)<d\left(\pi T_{s} x, T_{s} x\right)+\varepsilon \leq d\left(T_{\mu} x, T_{s} x\right)+\varepsilon$ for all $s \geqslant s_{0}$. Since $\left\{T_{s} x: s \in S\right\}$ is bounded by Lemma 3.2, there exists $M>0$ such that $d$ $\left(T_{\mu} x, T_{s} x\right)<M$ for all $s \in S$. Therefore, $d^{2}\left(P x, T_{s} x\right) \leq d^{2}\left(T_{\mu} x, T_{s} x\right)+2 M \varepsilon+\varepsilon^{2}$ for each $s$ $\geqslant s_{0}$. Since $\mu$ is an invariant mean, we have $\mu_{s}\left(d^{2}\left(P x, T_{s} x\right)\right)=\mu_{s}\left(d^{2}\left(P x, T_{s s_{0}} x\right)\right) \leq \mu_{s}\left(d^{2}\left(T_{\mu} x, T_{s s_{0}} x\right)\right)+2 M \varepsilon+\varepsilon^{2}=\mu_{s}\left(d^{2}\left(T_{\mu} x, T_{s} x\right)\right)+2 M \varepsilon+\varepsilon^{2}$ for any $\varepsilon$ $>0$. By the argminimality of $T_{\mu} x$ (see Lemma 2.2), $T_{\mu} x=P x$. $\square$

In order to obtain the Rodé's theorem (Theorem 1.1) in the framework of CAT(0) spaces, we need to restrict the asymptotically invariant nets of means $\left\{\mu_{\alpha}\right\}$ to those that satisfy an additional condition: for each $t \in S$,

$$
\mu_{\alpha_{s}}\left(d^{2}\left(T_{s} x, y\right)\right)-\mu_{\alpha_{s}}\left(d^{2}\left(T_{s t} x, y\right)\right) \rightarrow 0 \text { uniformly for } y \in C .
$$

In the Hilbert space setting, condition (3) is not required because the weak convergence can obtain from (2) directly.

Lemma 3.8. Let $X$ be a complete $C A T(0)$ space that has property (N), $C$ be a closed convex subset of $X, S$ a commutative semigroup, and $\mathcal{S}(S)$ a nonexpansive semigroup on $C$ with $F(\mathcal{S}) \neq \emptyset$. Suppose $\left\{\mu_{\alpha}\right\}$ is an asymptotically invariant nets of means on $B(S)$ satisfying condition (3). If $\left\{T_{\mu_{\alpha}} x\right\} \Delta$-converges to $x_{0}$, then $x_{0} \in F(\mathcal{S})$.

Proof. First, we show that, for each $r \in S$,

$$
\lim _{\alpha} d\left(T_{\mu_{\alpha}} x, T_{r} T_{\mu_{\alpha}} x\right)=0 .
$$

If this is not the case, there must be some $\delta>0$ so that for each $\alpha$, there exists $\alpha^{\prime}>$ $\alpha$ satisfying $d\left(T_{\mu_{\alpha^{\prime}}}, T_{r} T_{\mu_{\alpha^{\prime}}} x\right) \geq \delta$. Put $\varepsilon=\frac{\delta^{2}}{2}$. Since the asymptotically invariant net $\left\{\mu_{\alpha}\right\}$ satisfies (3), there exists $\alpha_{0}$ for which for each $\alpha \geqslant \alpha_{0}$, $\varphi_{\mu_{\alpha}}\left(T_{r} T_{\mu_{\alpha}} x\right)=\mu_{\alpha_{s}}\left(d^{2}\left(T_{s} x, T_{r} T_{\mu_{\alpha}} x\right)\right)<\mu_{\alpha_{s}}\left(d^{2}\left(T_{r} T_{s} x, T_{r} T_{\mu_{\alpha}} x\right)\right)+\varepsilon \leq \mu_{\alpha_{s}}\left(d^{2}\left(T_{s} x, T_{\mu_{\alpha}} x\right)\right)+\varepsilon=\varphi_{\mu_{\alpha}}\left(T_{\mu_{\alpha}} x\right)+\varepsilon_{.} \quad$ Set $w=\frac{\mathrm{T}_{\mu_{\alpha^{\prime}} 0} x \oplus T_{r} T_{\mu_{\alpha^{\prime} 0}} x}{2}$. By the (CN) inequality, the following in equalities hold for each $s \in S:$

$$
\begin{aligned}
d^{2}\left(T_{s} x, w\right) & \leq \frac{1}{2} d^{2}\left(T_{s} x, T_{\mu_{\alpha^{\prime} 0}} x\right)+\frac{1}{2} d^{2}\left(T_{s} x, T_{r} T_{\mu_{\alpha^{\prime} 0}} x\right)-\frac{1}{4} d^{2}\left(T_{\mu_{\alpha^{\prime} 0}} x, T_{r} T_{\mu_{\alpha^{\prime} 0}} x\right) \\
& \leq \frac{1}{2} d^{2}\left(T_{s} x, T_{\mu_{\alpha^{\prime} 0}} x\right)+\frac{1}{2} d^{2}\left(T_{s} x, T_{r} T_{\mu_{\alpha^{\prime} 0}} x\right)-\frac{\delta^{2}}{4} .
\end{aligned}
$$


Therefore,

$$
\begin{aligned}
\varphi_{\mu_{\alpha^{\prime} 0}}(w) & \leq \frac{1}{2} \varphi_{\mu_{\alpha^{\prime} 0}}\left(T_{\mu_{\alpha^{\prime} 0}} x\right)+\frac{1}{2} \varphi_{\mu_{\alpha^{\prime} 0}}\left(T_{r} T_{\mu_{\alpha^{\prime} 0}} x\right)-\frac{\delta^{2}}{4} \\
& <\varphi_{\mu_{\alpha^{\prime} 0}}\left(T_{\mu_{\alpha^{\prime} 0}} x\right)+\frac{\varepsilon}{2}-\frac{\delta^{2}}{4} \\
& =\varphi_{\mu_{\alpha^{\prime} 0}}\left(T_{\mu_{\alpha^{\prime} 0} 0} x\right),
\end{aligned}
$$

which is a contradiction and thus (4) holds.

Next, we show that $x_{0} \in F(\mathcal{S})$. We suppose on the contrary that $x_{0} \notin F(\mathcal{S})$. Thus, for some $r \in S, T_{r} x_{0} \neq x_{0}$, i.e., $d\left(x_{0}, T_{r} x_{0}\right):=\gamma>0$. Since $\left\{T_{\mu_{\alpha}} x\right\} \subset \overline{c o}\left\{T_{s} x\right\}$, it is bounded. We can get an $M>0$ so that $d\left(T_{\mu_{\alpha}} x, x_{0}\right) \leq M$ for all $\alpha$. We let $0<\varepsilon<\min \left\{\frac{\gamma^{2}}{16 M}, 2 M\right\}$. From (4), there exists $\alpha_{0}$ with the property that $d\left(T_{r} T_{\mu_{\alpha}} x, T_{\mu_{\alpha}} x\right)<\varepsilon$ for all $\alpha \geqslant \alpha_{0}$. Now, for each $\alpha \geqslant \alpha_{0}$, $d\left(T_{\mu_{\alpha}} x, T_{r} x_{0}\right) \leq d\left(T_{\mu_{\alpha}} x, T_{r} T_{\mu_{\alpha}} x\right)+d\left(T_{r} T_{\mu_{\alpha}} x, T_{r} x_{0}\right)<d\left(T_{\mu_{\alpha}} x, x_{0}\right)+\varepsilon$. Thus, $d^{2}\left(T_{\mu_{\alpha}} x, T_{r} x_{0}\right)<d^{2}\left(T_{\mu_{\alpha}} x, x_{0}\right)+2 \varepsilon d\left(T_{\mu_{\alpha}} x, x_{0}\right)+\varepsilon^{2}$. Let $w=\frac{x_{0} \oplus T_{r} x_{0}}{2}$. Using the (CN) inequality, we see that

$$
\begin{aligned}
d^{2}\left(T_{\mu_{\alpha}} x, w\right) & \leq \frac{1}{2} d^{2}\left(T_{\mu_{\alpha}} x, x_{0}\right)+\frac{1}{2} d^{2}\left(T_{\mu_{\alpha}} x, T_{r} x_{0}\right)-\frac{1}{4} d^{2}\left(x_{0}, T_{r} x_{0}\right) \\
& \leq \frac{1}{2} d^{2}\left(T_{\mu_{\alpha}} x, x_{0}\right)+\frac{1}{2}\left(d^{2}\left(T_{\mu_{\alpha}} x, x_{0}\right)+2 \varepsilon M+\varepsilon^{2}\right)-\frac{\gamma^{2}}{4} \\
& =d^{2}\left(T_{\mu_{\alpha}} x, x_{0}\right)+\varepsilon M+\frac{\varepsilon^{2}}{2}-\frac{\gamma^{2}}{4}
\end{aligned}
$$

for all $\alpha \geqslant \alpha_{0}$. Consequently,

$$
\begin{aligned}
\limsup _{\alpha} d^{2}\left(T_{\mu_{\alpha}} x, w\right) & \leq \limsup _{\alpha} d^{2}\left(T_{\mu_{\alpha}} x, x_{0}\right)+\varepsilon M+\frac{\varepsilon^{2}}{2}-\frac{\gamma^{2}}{4} \\
& <\limsup _{\alpha} d^{2}\left(T_{\mu_{\alpha}} x, x_{0}\right)
\end{aligned}
$$

contradicting to the fact that $\left\{x_{0}\right\}$ is the center of $\left\{T_{\mu_{\alpha}} x\right\}$. Therefore, $T_{r} x_{0}=x_{0}$ for all $r$ $\in S$, and this shows that $x_{0} \in F(\mathcal{S})$ as desired.

Theorem 3.9. Let $X$ be a complete $C A T(0)$ space that has Property $(N), C$ be a closed convex subset of $X, S$ a commutative semigroup, and $\mathcal{S}(S)$ a nonexpansive semigroup on $C$ with $F(\mathcal{S}) \neq \emptyset$. Suppose $\left\{\mu_{\alpha}\right\}$ is an asymptotically invariant net of means on $B(S)$ satisfying condition (3). Then, $\left\{T_{\mu_{\alpha}} x\right\} \Delta$-converges to Px $\in F(\mathcal{S})$ for all $x \in C$. Here, Px is defined in Proposition 3.6.

Proof. Let $x \in C$ and $\left\{\mu_{\alpha^{\prime}}\right\}$ be any subnet of $\left\{\mu_{\alpha}\right\}$. There exists a subnet $\left\{\mu_{\alpha^{\prime \prime}}\right\}$ of $\left\{\mu_{\alpha^{\prime}}\right\}$ such that $\left\{\mu_{\alpha^{\prime \prime}}\right\} \mathrm{w}^{*}$-converges to $\mu$ for some invariant mean $\mu$ on $B(S)$. By Proposition 3.7, $T_{\mu} x=P x$. Since the net $\left\{T_{\mu_{\alpha^{\prime \prime}}} x\right\} \overline{c o}\left\{T_{s} x: s \in S\right\}$, it is bounded. Then by Proposition 2.6, there exists a subnet $\left\{\mu_{\alpha_{\beta}}\right\}$ of $\left\{\mu_{\alpha^{\prime \prime}}\right\}$ such that $\left\{T_{\mu_{\alpha_{\beta}}} x\right\} \Delta$-converges to some $x_{0}$ $\in C$. By Lemma 3.8, $x_{0} \in F(\mathcal{S})$.

We show $x_{0}=T_{\mu} x$ by splitting the proof into three steps.

Step 1. If $\overline{T_{\mu_{\alpha_{\beta}}} x}:=\operatorname{argmin}\left\{y \mapsto \mu_{\beta_{s}}\left(d^{2}\left(T_{s s_{0}} x, y\right)\right)\right\}$, then $\overline{T_{\mu_{\alpha \beta}} x} \in \overline{c o}\left\{T_{s} x\right\}_{s \succcurlyeq s_{0}}$.

$$
\text { Suppose } \quad \overline{T_{\mu_{\alpha_{\beta}}} x} \notin \overline{c o}\left\{T_{s} x\right\}_{s \succcurlyeq s_{0}}, \quad \text { by }
$$

$d^{2}\left(T_{s s_{0}} x, \overline{T_{\mu_{\beta}} x}\right) \geq d^{2}\left(T_{s s_{0}} x, \pi \overline{T_{\mu_{\alpha_{\beta}}} x}\right)+d^{2}\left(\overline{T_{\mu_{\alpha_{\beta}}} x}, \pi \overline{T_{\mu_{\alpha_{\beta}}} x}\right)$ for each $s \in \quad S$ where $\pi: C \rightarrow \overline{c o}\left\{T_{s} x\right\}_{s \succcurlyeq s_{0}}$ is the nearest point projection. Thus, 
$\mu_{\alpha_{\beta}}\left(d^{2}\left(T_{s s_{0}} x, \overline{T_{\mu_{\alpha_{\beta}}} x}\right)\right) \geq \mu_{\alpha_{\beta}}\left(d^{2}\left(T_{s s_{0}} x, \pi \overline{T_{\mu_{\alpha_{\beta}}} x}\right)\right)+d^{2}\left(\overline{T_{\mu_{\alpha_{\beta}}} x}, \pi \overline{T_{\mu_{\alpha_{\beta}}} x}\right)>\mu_{\alpha_{\beta}}\left(d^{2}\left(T_{s s_{0}} x, \pi \overline{T_{\mu_{\alpha_{\beta}}} x}\right)\right)$. This impossibility shows that $\overline{T_{\mu_{\alpha \beta}} x} \in \overline{C 0}\left\{T_{s} x\right\}_{s \succcurlyeq s_{0}}$.

Step $2 . \lim _{\beta} d\left(T_{\mu_{\alpha_{\beta}}} x, \overline{T_{\mu_{\alpha_{\beta}}} x}\right)=0$.

If this does not hold, there must be some $\eta>0$ so that for each $\beta$, there exists $\beta^{\prime}>\beta$ satisfying $d\left(T_{\mu_{\alpha_{\beta^{\prime}}}} x, \overline{T_{\mu_{\beta^{\prime}}} x}\right) \geq \eta$. Put $\varepsilon=\frac{\eta^{2}}{2}$. Since the asymptotically invariant net $\left\{\mu_{\beta}\right\}$ satisfies (3), there exists $\beta_{0}$ such that $\left|\mu_{\alpha_{\beta_{s}}}\left(d^{2}\left(T_{s} x, \overline{T_{\mu_{\alpha_{\beta}}} x}\right)\right)-\mu_{\alpha_{\beta_{s}}}\left(d^{2}\left(T_{s s_{0}} x, \overline{T_{\mu_{\alpha_{\beta}}} x}\right)\right)\right|<\varepsilon$ for each $\beta \geqslant \beta_{0}$. We suppose first that $\mu_{\alpha_{\beta^{\prime} 0_{s}}}\left(d^{2}\left(T_{s s_{0}} x, \overline{T_{\mu_{\beta_{\beta^{\prime}} 0}} x}\right) \leq \mu_{\alpha_{\beta^{\prime} 0_{s}}}\left(d^{2}\left(T_{s} x, T_{\mu_{\alpha_{\beta^{\prime}} 0}} x\right)\right)\right.$. Set $w=\frac{T_{\mu_{\alpha_{\beta^{\prime}}{ }^{\prime}}} x \oplus \overline{T_{\mu_{\alpha_{\beta^{\prime} 0}}} x}}{2}$. By (CN) inequality, the following inequalities hold for each $s \in S$ :

$$
\begin{aligned}
d^{2}\left(T_{s} x, w\right) & \leq \frac{1}{2} d^{2}\left(T_{s} x, T_{\mu_{\alpha_{\beta^{\prime}}}} x\right)+\frac{1}{2} d^{2}\left(T_{s} x, \overline{T_{\mu_{\beta_{\beta^{\prime}} 0}} x}\right)-\frac{1}{4} d^{2}\left(T_{\mu_{\alpha_{\beta^{\prime} 0}}} x, \overline{T_{\mu_{\alpha_{\beta^{\prime}}}} x}\right) \\
& \leq \frac{1}{2} d^{2}\left(T_{s} x, T_{\mu_{\alpha_{\beta^{\prime}}}} x\right)+\frac{1}{2} d^{2}\left(T_{s} x, \overline{T_{\mu_{\alpha_{\beta^{\prime}}}} x}\right)-\frac{\eta^{2}}{4} .
\end{aligned}
$$

Therefore,

$$
\begin{aligned}
\varphi_{\mu_{\alpha_{\beta^{\prime} 0}}}(w) & \leq \frac{1}{2} \varphi_{\mu_{\alpha_{\beta^{\prime}} 0}}\left(T_{\mu_{\alpha_{\beta^{\prime}}}} x\right)+\frac{1}{2} \varphi_{\mu_{\alpha_{\beta^{\prime} 0}}}\left(\overline{T_{\mu_{\alpha_{\beta^{\prime} 0}}} x}\right)-\frac{\eta^{2}}{4} \\
& <\frac{1}{2} \varphi_{\mu_{\alpha_{\beta^{\prime} 0}}}\left(T_{\mu_{\alpha_{\beta^{\prime}} 0}} x\right)+\frac{1}{2} \mu_{\alpha_{\beta^{\prime} 0_{s}}}\left(d^{2}\left(T_{s s_{0}} x, \overline{T_{\mu_{\alpha_{\beta^{\prime}}}} x}\right)\right)+\frac{\varepsilon}{2}-\frac{\eta^{2}}{4} \\
& \leq \varphi_{\mu_{\alpha_{\beta^{\prime} 0}}}\left(T_{\mu_{\alpha_{\beta^{\prime}}}} x\right)+\frac{\varepsilon}{2}-\frac{\eta^{2}}{4}=\varphi_{\mu_{\alpha_{\beta^{\prime} 0}}}\left(T_{\mu_{\alpha_{\beta^{\prime} 0}}} x\right)
\end{aligned}
$$

contradicting to the argminimality of $T_{\mu_{\alpha_{\beta}^{\prime}}^{\prime}} x$. In case $\mu_{\alpha_{\beta^{\prime} 0_{s}}}\left(d^{2}\left(T_{s} x, T_{\mu_{\alpha_{\beta^{\prime}}}} x\right)\right)<\mu_{\alpha_{\beta^{\prime} 0_{s}}}\left(d^{2}\left(T_{s s_{0}} x, \overline{\left.T_{\mu_{\alpha_{\beta^{\prime}} 0}} x\right)}\right)\right.$, we can show in the same way that $\mu_{\alpha_{\beta^{\prime} 0_{s}}}\left(d^{2}\left(T_{s s_{0}} x, w\right)\right)<\mu_{\alpha_{\beta^{\prime} 0_{s}}}\left(d^{2}\left(T_{s s_{0}} x, \overline{T_{\mu_{\alpha_{\beta^{\prime}}}} x}\right)\right)$ for some $w$ which also leads to a contradiction.

Step 3. $x_{0}=T_{\mu} x$.

We suppose on the contrary and let $\eta:=d\left(x_{0}, T_{\mu} x\right)>0$. Let $I=\left[T_{\mu} x, x_{0}\right]$ and $\pi_{I}: C$ $\rightarrow I$ be the nearest point projection onto $I$. Since $\left\{T_{s} x\right\}$ is bounded, there exists $M>0$ such that $d\left(T_{s} x, \pi_{I}\left(T_{s} x\right)\right) \leq M$ for all $s \in S$. Set $N_{0}>\frac{4(M+\eta)}{5 \eta}$ and $\rho=\frac{\eta}{5 N_{0}}$. Suppose there exists $s_{0} \in S$ such that $d\left(\pi_{I}\left(T_{s} x\right), x_{0}\right) \geq 2 \rho$ for all $s \geqslant s_{0}$. We know, by Step 1 , that $\overline{T_{\mu_{\alpha_{\beta}}} x} \in \overline{c o}\left\{T_{s} x\right\}_{s \succcurlyeq s_{0}}$. Let $A:=\left\{y \in C: d\left(\pi_{I}(y), x_{0}\right)>2 \rho\right\}$. Using property $(\mathrm{N}), A$ is convex and $\overline{c o}\left\{T_{s} x\right\}_{s \succcurlyeq s_{0}} \subset \bar{A} \subset\left\{y \in C: d\left(\pi_{1}(y), x_{0}\right) \geq 2 \rho\right\}$ and thus $d\left(\pi_{I}\left(\overline{T_{\mu_{\alpha_{\beta}} x} x}\right), x_{0}\right) \geq 2 \rho$. By Step $2, \lim _{\beta} d\left(T_{\mu_{\alpha_{\beta}}} x, \overline{T_{\mu_{\alpha_{\beta}}} x}\right)=0$. Choose $\beta_{0}$, using the nonexpansiveness of $\pi_{I}$, so that $d\left(\pi_{I}\left(T_{\mu_{\alpha}} x\right), \pi_{I}\left(\overline{T_{\mu_{\alpha_{\beta}}} x}\right)\right)<\rho$ for all $\beta \geqslant \beta_{0}$. Thus, $d\left(\pi_{I}\left(T_{\mu_{\alpha_{\beta}}} x\right), x_{0}\right)>\rho$ for all $\beta \geqslant \beta_{0}$. But then $x_{0} \notin \overline{c o}\left\{T_{\mu_{\alpha_{\beta}}} x\right\}_{\beta \succcurlyeq \beta_{0}}$ which contradicts to the fact that $x_{0}$ is the $\Delta$ - limit of $\left\{T_{\mu_{\alpha \beta}} x\right\}$. Therefore, there must be a subnet $\left\{\mathrm{s}^{\prime}\right\}$ of $S$ such that $s^{\prime}>s$ for all $s$ and

$$
d\left(\pi_{I}\left(T_{s^{\prime}} x\right), x_{0}\right)<2 \rho=\frac{2 \eta}{5 N_{0}}
$$


for all $s^{\prime}$. Hence,

$$
d\left(\pi_{I}\left(T_{s^{\prime}} x\right), T_{\mu} x\right)=\eta-d\left(\pi_{I}\left(T_{s^{\prime}} x\right), x_{0}\right)>\eta-\frac{2 \eta}{5 N_{0}} .
$$

By the property of $N_{0}, 5 \eta^{2} N_{0}>4 \eta M+4 \eta^{2}$ and so

$$
\eta^{2}-\frac{4 \eta^{2}}{5 N_{0}}>\frac{4 \eta M}{5 N_{0}}
$$

From (5), (6), and (7),

$$
\begin{aligned}
d^{2}\left(\pi_{I}\left(T_{s^{\prime}} x\right), T_{\mu} x\right) & >\eta^{2}-\frac{4 \eta^{2}}{5 N_{0}}+\left(\frac{2 \eta}{5 N_{0}}\right)^{2}>\frac{4 \eta M}{5 N_{0}}+\left(\frac{2 \eta}{5 N_{0}}\right)^{2} \\
& >2 d\left(x_{0}, \pi_{I}\left(T_{s^{\prime}} x\right)\right) d\left(T_{s^{\prime}} x, \pi_{I}\left(T_{s^{\prime}} x\right)\right)+d^{2}\left(x_{0}, \pi_{I}\left(T_{s^{\prime}} x\right)\right) .
\end{aligned}
$$

Using (1),

$$
\begin{aligned}
d^{2}\left(T_{s^{\prime}} x, T_{\mu} x\right) & \geq d^{2}\left(\pi_{I}\left(T_{s^{\prime}} x\right), T_{\mu} x\right)+d^{2}\left(\pi_{I}\left(T_{s^{\prime}} x\right), T_{s^{\prime}} x\right) \\
& >d^{2}\left(x_{0}, \pi_{I}\left(T_{s^{\prime}} x\right)\right)+2 d\left(x_{0}, \pi_{I}\left(T_{s^{\prime}} x\right)\right) d\left(T_{s^{\prime}} x, \pi_{I}\left(T_{s^{\prime}} x\right)\right)+d^{2}\left(\pi_{I}\left(T_{s^{\prime}} x\right), T_{s^{\prime}} x\right) \\
& =\left(d\left(x_{0}, \pi_{I}\left(T_{s^{\prime}} x\right)\right)+d\left(T_{s^{\prime}} x, \pi_{I}\left(T_{s^{\prime}} x\right)\right)\right)^{2} \\
& \geq d^{2}\left(T_{s^{\prime}} x, x_{0}\right)
\end{aligned}
$$

for all $s^{\prime}$. Since the points $x_{0}$ and $T_{\mu} x$ belong to the set $F(\mathcal{S})$, the nets $\left\{d^{2}\left(T_{s} x, x_{0}\right)\right\}$ and $\left\{d^{2}\left(T_{s} x, T_{\mu} x\right)\right\}$ are decreasing. So, $\lim _{s} d^{2}\left(T_{s} x, x_{0}\right)$ and $\lim _{s} d^{2}\left(T_{s} x, T_{\mu} x\right)$ exist. Hence, $\phi_{\mu}\left(T_{\mu} x\right)=\lim _{s} d^{2}\left(T_{s} x, T_{\mu} x\right)=\lim _{s^{\prime}} d^{2}\left(T_{s^{\prime}} x, T_{\mu} x\right)=\mu_{s^{\prime}}\left(d^{2}\left(T_{s^{\prime}} x, T_{\mu} x\right)\right) \geq \mu_{s^{\prime}}\left(d^{2}\right.$ $\left.\left(T_{s^{\prime}} x, x_{0}\right)\right)=\lim _{s^{\prime}} d^{2}\left(T_{s^{\prime}} x, x_{0}\right)=\lim _{s} d^{2}\left(T_{s} x, x_{0}\right)=\phi_{\mu}\left(x_{0}\right)$, a contradiction. Thus, $x_{0}=$ $T_{\mu} x$.

The above argument shows that, for every subnet $\left\{\mu_{\alpha^{\prime}}\right\}$ of $\left\{\mu_{\alpha}\right\}$, there exists a subnet $\left\{\mu_{\alpha_{\beta}}\right\}$ of $\left\{\mu_{\alpha}\right\}$ such that $\left\{T_{\mu_{\alpha_{\beta}}} x\right\} \Delta$-converges to $T_{\mu} x(=P x)$. By Remark 2.7 (ii), $\left\{T_{\mu_{\alpha}} x\right\}$ $\Delta$-converges to $P$ x.

It is an interesting open problem to determine whether Theorem 3.9 remains valid when the semigroup is amenable but not commutative.

\subsection{Applications}

Proposition 3.10. Let $C$ be a closed convex subset of a complete $C A T(0)$ space $X$ and $T$ $: C \rightarrow C$ be a nonexpansive mapping with $F(T) \neq \varnothing$. Let $S=(\mathbb{N} \cup\{0\},+)$, $\mathcal{S}(S)=\left\{T^{n}: n \in S\right\}, \Lambda=\mathbb{N}$ or $\mathbb{R}^{+}$and $\beta_{\lambda k} \geq 0$ be such that $\sum_{k \in S} \beta_{\lambda k}=1$ for all $\lambda \in \Lambda$. Suppose for all $k \in S$,

$$
\lim _{\lambda \rightarrow \infty} \beta_{\lambda k}=0
$$

and for each $m \in S$,

$$
\lim _{\lambda \rightarrow \infty} \sum_{k=m}^{\infty}\left|\beta_{\lambda k}-\beta_{\lambda(k-m)}\right|=0 .
$$

For any $f=\left(a_{0}, a_{1}, \ldots\right) \in B(S)$ let $\mu_{\lambda}(f)=\sum_{k=0}^{\infty} \beta_{\lambda k} a_{k}$. Then for each $x \in C$, $\left\{T_{\mu_{\lambda}} x\right\} \Delta$-converges to $z$ for some $z$ in $F(T)$.

In particular, if $X$ is a Hilbert space, we have $\sum_{k=0}^{\infty} \beta_{\lambda k} T^{k} x c o n v e r g e s$ weakly to $z$ for some $z$ in $F(T)$ as $\lambda \rightarrow \infty$. 
Proof. For each $m \in S,\left|\mu_{\lambda}(f)-\mu_{\lambda}\left(r_{m} f\right)\right|=\left|\sum_{k=0}^{\infty} \beta_{\lambda k} a_{k}-\sum_{k=0}^{\infty} \beta_{\lambda k} a_{k+m}\right| \leq \sum_{k=0}^{m-1}\left|\beta_{\lambda k}\right|\left|a_{k}\right|+\sum_{k=m}^{\infty}\left|\beta_{\lambda k}-\beta_{\lambda(k-m)}\right|\left|a_{k}\right|$. By (8) and (9), we have $\lim _{\lambda \rightarrow \infty}\left|\mu_{\lambda}(f)-\mu_{\lambda}\left(r_{m} f\right)\right|=0$, and this shows that the net $\left\{\mu_{\lambda}\right\}$ is asymptotically invariant. Let $x \in C$ and consider $a_{k}$ of the form $a_{k}=d^{2}\left(T^{k} x, y\right)$ where $y \in$ $C$. We see that $\left\{\mu_{\lambda}\right\}$ satisfies (3). By Theorem 3.9, we have $\left\{T_{\mu_{\lambda}} x\right\} \Delta$ - converges to $z$ for some $z$ in $F(T)$.

In Hilbert spaces, by a well-known result in probability theory, we know that

$$
\sum_{k=0}^{\infty} \beta_{\lambda k}\left\|T^{k} x-\sum_{k=0}^{\infty} \beta_{\lambda k} T^{k} x\right\|^{2} \leq \sum_{k=0}^{\infty} \beta_{\lambda k}\left\|T^{k} x-y\right\|^{2}
$$

for all $y \in C$. So we have $T_{\mu_{\lambda}} x=\sum_{k=0}^{\infty} \beta_{\lambda k} T^{k} x$. $\square$

Corollary 3.11 (Bailon Ergodic Theorem). Let $C$ be a closed convex subset of a Hilbert space $H$ and $T: C \rightarrow C$ be a nonexpansive mapping with $F(T) \neq \varnothing$. Then, for any $x \in C$,

$$
S_{n} x=\frac{1}{n} \sum_{k=0}^{n-1} T^{k} x
$$

converges weakly to $z$ for some $z$ in $F(T)$ as $n \rightarrow \infty$.

Proof. Let $\Lambda=\mathbb{N}$ and put, for $\lambda \in \Lambda$ and $k \in S=(\mathbb{N} \cup\{0\},+)$,

$$
\beta_{\lambda k}=\left\{\begin{array}{l}
\frac{1}{\lambda}, k \leq \lambda-1 \\
0, k>\lambda-1
\end{array}\right.
$$

The result now follows from Proposition 3.10. $\square$

Corollary 3.12. [[15], Theorem 3.5.1] Let $C$ be a closed convex subset of a Hilbert space $H$ and $T: C \rightarrow C$ be a nonexpansive mapping with $F(T) \neq \varnothing$. Then, for any $x \in$ $C, S_{r} x=(1-r) \sum_{k=0}^{\infty} r^{k} T^{k} x$ converges weakly to $z$ for some $z$ in $F(T)$ as $r \uparrow 1$.

Proof. Let $\Lambda=\mathbb{R}^{+}$and put, for $\lambda \in \Lambda$ and $k \in S=(\mathbb{N} \cup\{0\},+)$,

$$
\beta_{\lambda k}=\frac{(\lambda-1)^{k}}{\lambda^{k+1}} .
$$

Taking $r=\frac{\lambda-1}{\lambda}$, Proposition 3.10 implies the desired result.

Let $S=\left(\mathbb{R}^{+} \cup\{0\},+\right)$ and $C$ be a closed convex subset of a Hilbert space $H$. Then, a family $\mathcal{S}(S)=\{T(s): s \in S\}$ is said to be a continuous nonexpansive semigroup on $C$ if $\mathcal{S}(S)$ satisfies the following:

(i) $T(s): C \rightarrow C$ is a nonexpansive mapping for all $s \in S$,

(ii) $T(t+s) x=T(t) T(s) x$ for all $x \in C$ and $t, s \in S$,

(iii) for each $x \in C$, the mapping $s \rightarrow T(s) x$ is continuous, and

(iv) $T(0) x=x$ for all $x \in C$.

Proposition 3.13. Let $C$ be a closed convex subset of a Hilbert space $H$. Let $S=\left(\mathbb{R}^{+} \cup\right.$ $\{0\},+), \mathcal{S}(S)$ be a continuous nonexpansive semigroup on $C$ with $F(\mathcal{S}) \neq \emptyset, \Lambda=\mathbb{R}^{+}$and $g_{\lambda}$ be a density function on S,i.e., $g_{\lambda} \geq 0$ and $\int_{0}^{\infty} g_{\lambda}(s) d s=1$ for all $\lambda \in \Lambda$. Suppose $g_{\lambda}$ satisfies the following properties. for each $h \in S$, 


$$
\lim _{\lambda \rightarrow \infty} g_{\lambda}(s)=0
$$

uniformly on $[0, h]$ and

$$
\lim _{\lambda \rightarrow \infty} \int_{h}^{\infty}\left|g_{\lambda}(s)-g_{\lambda}(s-h)\right| d s=0 .
$$

Then, for any $x \in C$,

$$
\int_{0}^{\infty} g_{\lambda}(s) T(s) x d s
$$

converges weakly to some $z \in F(\mathcal{S})$ as $\lambda \rightarrow \infty$.

Proof. For $f \in B(S)$ we define $\mu_{\lambda}(f)=\int_{0}^{\infty} g_{\lambda}(s) f(s) d s$ for all $\lambda>0$. Thus, $\mu_{\lambda}$ is a mean on $B(S)$. For any $h \in S$ we consider

$$
\begin{aligned}
\left|\mu_{\lambda}(f)-\mu_{\lambda}\left(r_{h} f\right)\right| & =\left|\int_{0}^{\infty} g_{\lambda}(s) f(s) d s-\int_{0}^{\infty} g_{\lambda}(s) f(s+h) d s\right| \\
& \leq \int_{0}^{h}\left|g_{\lambda}(s)\right||f(s)| d s+\int_{h}^{\infty}\left|g_{\lambda}(s)-g_{\lambda}(s-h)\right||f(s)| d s .
\end{aligned}
$$

By (10) and (11), $\lim _{\lambda}\left|\mu_{\lambda}(f)-\mu_{\lambda}\left(r_{h} f\right)\right|=0$. So, $\left\{\mu_{\lambda}\right\}$ is asymptotically invariant. For each $z \in C$, let $f(s)=\|z-T(s) x\|^{2}$. We see that $\left\{\mu_{\lambda}\right\}$ satisfies (3). For each $x \in C$, we know that

$$
\int_{0}^{\infty} g_{\lambda}(s)\left\|\int_{0}^{\infty} g_{\lambda}(s) T(s) x d s-T(s) x\right\|^{2} d s \leq \int_{0}^{\infty} g_{\lambda}(s)\|\gamma-T(s) x\|^{2} d s
$$

for all $y \in C$. Thus, $T_{\mu_{\lambda}} x=\int_{0}^{\infty} g_{\lambda}(s) T(s) x d s$. By Theorem 3.9, we have $\int_{0}^{\infty} g_{\lambda}(s) T(s) x d s$ converges weakly to some $z \in F(\mathcal{S})$ as $\lambda \rightarrow \infty$. $\square$

Corollary 3.14. [[15], Theorem 3.5.2] Let $C$ be a closed convex subset of a Hilbert space $H$. Suppose $S=\left(\mathbb{R}^{+} \cup\{0\},+\right)$ and $\mathcal{S}(S)$ be a continuous nonexpansive semigroup on $C$ with $F(\mathcal{S}) \neq \emptyset$. Then, for any $x \in C$,

$$
S_{\lambda} x=\frac{1}{\lambda} \int_{0}^{\lambda} T(s) x d s
$$

converges weakly to some $z \in F(\mathcal{S})$ as $\lambda \rightarrow \infty$.

Proof. Let $\Lambda=\mathbb{R}^{+}$and put, for $\lambda \in \Lambda$ and $s \in S, g_{\lambda}(s)=\frac{1}{\lambda} \chi_{[0, \lambda]}$. The result now follows from Proposition 3.13.

Corollary 3.15. [[15], Theorem 3.5.3] Let $C$ be a closed convex subset of a Hilbert space $H$. Suppose $S=\left(\mathbb{R}^{+} \cup\{0\},+\right)$ and $\mathcal{S}(S)$ be a continuous nonexpansive semi-group on $C$ with $F(\mathcal{S}) \neq \emptyset$. Then, for any $x \in C$,

$$
r \int_{0}^{\infty} e^{-r s} T(s) x d s
$$

converges weakly to some $z \in F(\mathcal{S})$ as $r \downarrow 0$.

Proof. Let $\Lambda=\mathbb{R}^{+}$and put, for $\lambda \in \Lambda$ and $s \in S, g_{\lambda}(s)=\frac{1}{\lambda} e^{-\frac{1}{\lambda} s}$. Again, we can then apply Proposition 3.13 by taking $r=\frac{1}{\lambda}$. $\square$ 
By using Lemma 2.2, we can obtain a strong convergence theorem in Hilbert spaces stated as Theorem 3.17 below.

Proposition 3.16. Let $C$ be a closed convex subset of a Hilbert space $H$ and $T: C \rightarrow$ $C$ be a nonexpansive mapping with $F(T) \neq \varnothing$. Given $x \in C$ and let $r=r\left(C,\left\{T^{n} x\right\}\right)$. Let $z$ be the unique asymptotic center of $\left\{T^{n} x\right\}$. For each $n \in \mathbb{N}$, define

$$
\Pi_{n}:=\left\{p=\left\{\beta_{n k}\right\}_{k \geq n} \subset[0,1]: \sum_{k \geq n} \beta_{n k}=1\right\}
$$

and

$$
V_{n}:=\sup _{p \in \Pi_{n}} \sum_{k \geq n} \beta_{n k}\left\|T^{k} x-\bar{x}_{n}\right\|^{2}
$$

where $\bar{x}_{n}=\sum_{k \geq n} \beta_{n k} T^{k} x$. If $V:=\lim _{n \rightarrow \infty} V_{n}$, then $V=r^{2}$.

Proof. Given $\varepsilon>0$. Since $z \in A\left(C,\left\{T^{n} x\right\}\right)$, by Remark 2.4 (ii), $z \in F(T)$. Choose $n_{\varepsilon} \in \mathbb{N}$ such that $\| T^{n} x-z||<r+\varepsilon$ for all $n \geq n_{\varepsilon}$. Fix $n \geq n_{\varepsilon}$ and let $p=\left\{\beta_{n k}\right\}_{k \geq n} \in \Pi_{n}$. Thus,

$$
\sum_{k \geq n} \beta_{n k}\left\|T^{k} x-\bar{x}_{n}\right\|^{2} \leq \sum_{k \geq n} \beta_{n k}\left\|T^{k} x-z\right\|^{2}<(r+\varepsilon)^{2} .
$$

So $V_{n}=\sup _{p \in \Pi_{n}} \sum_{k \geq n} \beta_{n k}\left\|T^{k} x-\bar{x}_{n}\right\|^{2}<(r+\varepsilon)^{2}$. Letting $n \rightarrow \infty, V=\lim _{n} \rightarrow \infty V_{n} \leq$ $(r+\varepsilon)^{2}$ for any $\varepsilon>0$. Hence, $V \leq r^{2}$.

Next, we show that $r^{2} \leq V$. Indeed, since $z \in \overline{c o}\left\{T^{k} x\right\}_{k \geq n}$ for all $n \in \mathbb{N}$, there exists a sequence $\left\{\bar{x}_{n}\right\}$ with $\bar{x}_{n} \in \operatorname{co}\left\{T^{k} x\right\}_{k \geq n}$ for each $n$ and $\bar{x}_{n} \rightarrow z$ as $n \rightarrow \infty$. Put $\bar{x}_{n}=\sum_{k \geq n} \beta_{n k} T^{k} x$. Since $\left\{T^{n} x\right\}$ is bounded, there exists $M>0$ such that $\left\|T^{k} x-\bar{x}_{n}\right\|+\left\|T^{k} x-z\right\| \leq M$. For each $\varepsilon>0$, choose $n_{\varepsilon} \in \mathbb{N}$ such that $\left\|\bar{x}_{n}-z\right\|<\varepsilon, V_{n}$ $<V+\varepsilon$ for all $n \geq n_{\varepsilon}$, and $\left\|T^{k} x-z\right\|>r-\varepsilon$ for all $k \geq n_{\varepsilon}$. Thus for any $n \geq n_{\varepsilon}$,

$$
\begin{aligned}
\sum_{k \geq n} \beta_{n k} \mid\left\|T^{k} x-\bar{x}_{n}\right\|^{2} & -\left\|T^{k} x-z\right\|^{2} \mid \\
& =\sum_{k \geq n} \beta_{n k}\left\|T^{k} x-\bar{x}_{n}\right\|-\left\|T^{k} x-z\right\| \|\left(\left\|T^{k} x-\bar{x}_{n}\right\|+\| T^{k} x-z \mid\right) \\
& =\sum_{k \geq n} \beta_{n k}|| \bar{x}_{n}-z \|\left(\left\|T^{k} x-\bar{x}_{n}\right\|+\left\|T^{k} x-z\right\|\right) \leq \varepsilon M .
\end{aligned}
$$

Hence,

$$
\begin{aligned}
(r-\varepsilon)^{2} & <\sum_{k \geq n} \beta_{n k}\left\|T^{k} x-z\right\|^{2} \\
& =\sum_{k \geq n} \beta_{n k}\left\|T^{k} x-z\right\|^{2}+\sum_{k \geq n} \beta_{n k}\left\|T^{k} x-\bar{x}_{n}\right\|^{2}-\sum_{k \geq n} \beta_{n k}\left\|T^{k} x-\bar{x}_{n}\right\|^{2} \\
& \leq V_{n}+\sum_{k \geq n} \beta_{n k}\left\|T^{k} x-\bar{x}_{n}\right\|^{2}-\left\|T^{k} x-z\right\|^{2} \mid<V+\varepsilon+\varepsilon M .
\end{aligned}
$$

So $(r-\varepsilon)^{2}<V+\varepsilon+\varepsilon M$ for any $\varepsilon>0$. This implies $r^{2} \leq V$.

Theorem 3.17. Let $C$ be a closed convex subset of a Hilbert space $H$ and $T: C \rightarrow C$ be a nonexpansive mapping with $F(T) \neq \varnothing$. Suppose $z, \Pi_{n}, V$, and $\bar{x}_{n}$ be defined as in Proposition 3.16. If the sequence $\left\{\bar{x}_{n}\right\}$ satisfies 


$$
\lim _{n \rightarrow \infty}\left(\sum_{k \geq n} \beta_{n k}\left\|T^{k} x-\bar{x}_{n}\right\|^{2}\right)=V,
$$

then $\left\{\bar{x}_{n}\right\}$ converges (strongly) to $z \in F(T)$ as $n \rightarrow \infty$.

Proof. Suppose for some $\varepsilon>0$, there exists a subsequence $\left\{\bar{x}_{n_{l}}\right\}$ of $\left\{\bar{x}_{n}\right\}$ such that $\left\|\bar{x}_{n_{l}}-z\right\| \geq \varepsilon$ for all $l \in \mathbb{N}$. For each $y \in C$ and $n \in \mathbb{N}$, define $\varphi_{n}(y):=\sum_{k \geq n} \beta_{n k}\left\|T^{k} x-y\right\|^{2}$. Let $0<\delta<\frac{\varepsilon^{2}}{8}$. By (12) and $z \in A\left(C,\left\{T^{k} x\right\}\right)$, we choose $n_{\delta}$ such that $r^{2}-\delta=V-\delta<\varphi_{n_{l}}\left(\bar{x}_{n_{l}}\right)<V+\delta=r^{2}+\delta$ for all $n_{l} \geq n_{\delta}$ and $\left\|T^{k} x-z\right\|^{2}$ $<r^{2}+\delta$ for all $k \geq n_{\delta}$. Fix $l \geq n_{\delta}$ and let $\omega=\frac{\bar{x}_{n_{l}}+z}{2}$. By the Parallelogram law, we have for each $k \geq n_{l}$,

$$
\left\|T^{k} x-\omega\right\|^{2}=\frac{1}{2}\left\|T^{k} x-\bar{x}_{n_{l}}\right\|^{2}+\frac{1}{2}\left\|T^{k} x-z\right\|^{2}-\frac{1}{4}\left\|\bar{x}_{n_{l}}-z\right\|^{2} .
$$

Hence,

$$
\varphi_{n_{l}}(\omega)<\frac{1}{2}\left(r^{2}+\delta\right)+\frac{1}{2}\left(r^{2}+\delta\right)-\frac{1}{4} \varepsilon^{2}<r^{2}-\delta<\varphi_{n_{l}}\left(\bar{x}_{n_{l}}\right) .
$$

Using Lemma 2.2, we see that this contradicts to the minimality of $\varphi_{n_{l}}\left(\bar{x}_{n_{l}}\right)$. $\square$

\section{Acknowledgements}

The authors would like to thank Anthony To-Ming Lau for drawing the problem into our attention and also for giving valuable advice during the preparation of the manuscript. We thank the referee for valuable and useful comments. We also wish to thank the National Research University Project under Thailand's Office of the Higher Education Commission for financial support.

\section{Author details}

${ }^{1}$ Department of Mathematics, Faculty of Science, Chiang Mai University, Chiang Mai, 50200, Thailand ${ }^{2}$ Materials Science Research Center, Faculty of Science, Chiang Mai University, Chiang Mai, 50200, Thailand

\section{Authors' contributions}

All authors read and approved the final manuscript.

\section{Competing interests}

The authors declare that they have no competing interests.

Received: 1 February 2011 Accepted: 15 August 2011 Published: 15 August 2011

\section{References}

1. Lim, TC: Remarks on some fixed point theorems. Proc Am Math Soc. 60, 179-182 (1976). doi:10.1090/S0002-9939-19760423139-X

2. Kirk, WA, Panyanak, B: A concept of convergence in geodesic spaces. Nonlinear Anal. 68, 3689-3696 (2008). doi:10.1016/ j.na.2007.04.011

3. Day, MM: Amenable semigroups. Illinois J Math. 1, 509-544 (1957)

4. Rodé, G: An ergodic theorem for semigroups of nonexpansive mappings in a Hilbert space. J Math Anal Appl. 85 172-178 (1982). doi:10.1016/0022-247X(82)90032-4

5. Kaniuth, E, Lau, AT, Pym, J: On character amenability of Banach algebras. J Math Anal Appl. 34, $942-955$ (2008)

6. Kada, O, Lau, AT, Takahashi, W: Asymptotically invariant nets and fixed point sets for semigroups of nonexpansive mappings. Nonlinear Anal. 29, 539-550 (1997). doi:10.1016/S0362-546X(96)00063-6

7. Lau, AT: Semigroup of nonexpansive mappings on a Hilbert space. J Math Anal Appl. 105, 514-522 (1985). doi:10.1016/ 0022-247X(85)90066-6

8. Lau, AT, Shioji, N, Takahashi, W: Existence of nonexpansive retractions for amenable semigroups of nonexpansive mappings and nonlinear ergodic theorems in Banach spaces. J Funct Anal. 191, 62-75 (1999)

9. Bridson, M, Haeiger, A: Metric Spaces of Non-Positive Curvature. Springer, Berlin (1999)

10. Sturm, KT: Probability measures on metric spaces of non-positive curvature. Heat Kernels and Analysis on Manifolds, Graphs and Metric Spaces (Paris, 2002), Contemp Math 338, Am Math Soc Providence, Rl. 357-390 (2003)

11. Espínola, R, Fernández-León, A: CAT(K) spaces, weak convergence and fixed points. J Math Anal Appl. 353, 410-427 (2009). doi:10.1016/j.jmaa.2008.12.015

12. Kakavandi, BA, Amini, M: Non-linear ergodic theorem in complete non-positive curvature metric spaces. Bull Iran Math Soc. (in press) 
13. Berg, ID, Nikolaev, IG: Quasilinerization and curvature of Alexandrov spaces. Geom Dedicata. 133, 195-218 (2008). doi:10.1007/s10711-008-9243-3

14. Kakavandi, BA, Amini, M: Duality and subdifferential for convex functions on complete CAT(0) metric spaces. Nonlinear Anal. 73, 3450-3455 (2010). doi:10.1016/..na.2010.07.033

15. Takahashi, W: Nonlinear Functional Analysis. Yokohama Publisher, Yokohama (2000)

doi:10.1186/1687-1812-2011-34

Cite this article as: Anakkamatee and Dhompongsa: Rodé's theorem on common fixed points of semigroup of nonexpansive mappings in CAT(0) spaces. Fixed Point Theory and Applications 2011 2011:34.

Submit your manuscript to a SpringerOpen ${ }^{\odot}$ journal and benefit from:

- Convenient online submission

- Rigorous peer review

- Immediate publication on acceptance

- Open access: articles freely available online

- High visibility within the field

- Retaining the copyright to your article

Submit your next manuscript at $\boldsymbol{\nabla}$ springeropen.com 